\title{
Association between Use of Statin and Risk of Dementia: A Meta-Analysis of Observational Studies
}

\author{
Tahmina Nasrin Poly a, b, g Md. Mohaimenul Islam ${ }^{a, b, g}$ Bruno Andreas Walther ${ }^{f}$ \\ Hsuan-Chia Yang a, b,g Chieh-Chen Wu ${ }^{a}$ Ming-Chin Lin ${ }^{\text {a,e }}$ Yu-Chuan Li ${ }^{a-d, g}$ \\ ${ }^{a}$ Graduate Institute of Biomedical Informatics, College of Medical Science and Technology, Taipei Medical \\ University, Taipei, Taiwan; ${ }^{b}$ International Center for Health Information Technology, Taipei Medical University, \\ Taipei, Taiwan; ' Department of Dermatology, Wan Fang Hospital, Taipei, Taiwan; ${ }^{\mathrm{d}}$ TMU Research Center of \\ Cancer Translational Medicine, Taipei, Taiwan; 'Department of Neurosurgery, Shuang Ho Hospital, Taipei Medical \\ University, Taipei, Taiwan; ${ }^{f}$ Department of Biological Sciences, National Sun Yat-sen University, Kaohsiung, Taiwan; \\ ${ }^{g}$ Research Center of Big Data and Meta-analysis, Wan Fang Hospital, Taipei Medical University, Taipei, Taiwan
}

\section{Keywords}

Statin · Dementia · Alzheimer · Vascular dementia · Cardiac disease $\cdot$ Meta-analysis

\begin{abstract}
Background and Aims: The impact of statin on dementia risk reduction has been a subject of debate over the last decade, but the evidence remains inconclusive. Therefore, we performed a meta-analysis of relevant observational studies to quantify the magnitude of the association between statin therapy and the risk of dementia. Methods: We systematically searched for relevant studies published from January 2000 to March 2018 using EMBASE, Google, Google Scholar, PubMed, Scopus, and Web of Science. Two authors performed study selection, data abstraction, and risk of bias assessment. We then extracted data from the selected studies and performed meta-analysis of observational studies using a random-effects model. Subgroup and sensitivity analyses were also conducted. Results: A total of 30 observational studies, including 9,162,509 participants (84,101 dementia patients), met the eligibility criteria. Patients with statin had a lower all-caused dementia risk than those without statin (risk ratio $[\mathrm{RR}] 0.83,95 \% \mathrm{Cl} 0.79-0.87, \mathrm{I}^{2}=57.73 \%$ ). The over-
\end{abstract}

all pooled reduction of Alzheimer disease in patients with statin use was RR 0.69 (95\% Cl 0.60-0.80, $p<0.0001)$, and the overall pooled RR of statin use and vascular dementia risk was RR 0.93 (95\% Cl 0.74-1.16, $p=0.54$ ). Conclusion: This study suggests that the use of statin is significantly associated with a decreased risk of dementia. Future studies measuring such outcomes would provide useful information to patients, clinicians, and policymakers. Until further evidence is established, clinicians need to make sure that statin use should remain restricted to the treatment of cardiovascular disease.

(c) 2019 S. Karger AG, Base

\section{Introduction}

Dementia is the most common neurological disorder, which is characterized by a decline in memory and other thinking skills. Its prevalence has been increasing steadily due to dramatic increases in life expectancy, imposing immense economic and social burdens [1]. Approximately 46.8 million people worldwide were afflicted with dementia in 2015, and this number is expected to reach 131.5 million by 2050 [2]. Several studies suggested that oxidative

\section{KARGER}

(c) 2019 S. Karger AG, Basel

karger@karger.com

www.karger.com/ned
Prof. Yu-Chuan Li, MD, PhD

Department of Dermatology

Wan Fang Hospital

$250 \mathrm{Wu}-\mathrm{Hsing}$ Street, Taipei 110 (Taiwan)

E-Mail jack@ tmu.edu.tw or jaak88@gmail.com 
stress, senile plaques, vascular impairment, cerebrovascular atherosclerosis, $\beta$-amyloid, neurofibrillary tangles, and hyperphosphorylated tau protein accumulation could be responsible for developing dementia $[3,4]$.

Statin is a HMG-CoA ( $\beta$-hydroxy $\beta$-methylglutaryl$\mathrm{CoA}$ ) reductase inhibitor that oppresses the pace of cholesterol synthesis in the liver, and it is widely used to treat cardiovascular disease. These medications have a host of pleiotropic effects and assist to inhibit the inflammation process, improve vascular flow, and suppress the production of reactive oxygen species [5]. A laboratory study using mice showed that simvastatin attenuates oxidative stress and inflammation in the amyloid precursor protein [6]. Another animal study investigating Alzheimer disease $(\mathrm{AD})$ reported that fluvastatin helps to mitigate oxidative damage and ameliorate neural degeneration and cognitive dysfunction in rats [7]. However, dysfunction of neurovascular coupling is thought to be involved in the dementia risk by impairing cerebral blood flow in response to proper neural function [8]. HMG-CoA reductase inhibitors help to reverse neurovascular dysfunction coupling by assuaging oxidative stress and inflammation [9]. Due to these assumed effects of statin medications on preventing or slowing dementia, epidemiological studies have already been conducted which report on their effectiveness. These studies could play an important role in the clinical decision-making process if we can generalize these findings from different kinds of population.

A previous meta-analysis by Zhou et al. [10] demonstrated that statin use did not have a beneficial effect on the risk of dementia or AD. A systematic review and meta-analysis by Song et al. [11] observed that 6 out of 8 studies reported a decreased risk of dementia in patients using statins; they nevertheless argued that causality remained inconclusive. Their findings were only stratified by follow-up duration and number of dementia cases. Furthermore, Wong et al. [12] included 20 studies in their meta-analysis which suggested that statins might provide a slight benefit in the prevention of $\mathrm{AD}$ and alltype dementia. Meanwhile, Rojas-Fernandez et al. [13] reported that statin use was not associated with dementia risk. A meta-analysis of 25 studies by Chu et al. [14] mentioned that the use of statins may reduce the risk of alltype dementia (adjusted risk ratio [aRR] 0.849, 95\% CI 0.787-0.916), $\mathrm{AD}$ (aRR 0.719, 95\% CI 0.576-0.899), and mild cognitive impairment ([aRR 0.737, 95\% CI 0.556-0.976]), but not of incident vascular dementia (VaD; [aRR 1.012, 95\% CI 0.620-1.652]). However, the findings of these meta-analyses did not consider different duration and individual statin use.

Statin Use and Risk of Dementia
Since the impact of statins on dementia risk reduction remains the subject of an ongoing debate, an updated meta-analysis with a broadened capacity for testing hypotheses can potentially yield more robust estimates $[15,16]$. Large number of studies with incidence cases might strengthen the statistical power of meta-analysis to evaluate the magnitude of the association between exposure and outcome risk. In our meta-analysis, we therefore aimed to synthesize the available evidence to estimate the association of dementia risk and statin use based on gender, continents, individual statin use, and duration of statin use. We also tried to review the biological evidence to support our findings.

\section{Methods}

We performed a systematic review of the literature according to Preferred Reporting Items for Systematic Reviews and MetaAnalyses based on the Cochrane systematic review and meta-analysis guidelines. We have previously used this method in several published reviews [17-19].

\section{Literature Review}

Electronic Searches

We conducted a literature search to identify all potential published and unpublished observational studies. The literature search identified eligible studies published in English only. The following electronic databases were used to identify potential studies:

(i) EMBASE (January 2000 and March 2018);

(ii) MEDLINE (January 2000 and March 2018);

(iii) Scopus (January 2000 and March 2018);

(iv) Google Scholar (January 2000 and March 2018);

(v) PubMed (January 2000 and March 2018);

Searching Other Resources

We also checked the reference lists of all the primary studies and review articles. We also conducted a search of ClinicalTrials. gov (www.clinicaltrials.gov).

\section{Search Terms}

The search terms used were "statin," "lipid lowering drugs," "cholesterol lowering drugs," "simvastatin," "atorvastatin," "pravastatin," "fluvastatin," "lovastatin," "observational study," "case-control study," "dementia," "AD," and "VaD."

Eligibility Criteria

We only included studies if they fulfilled the following PICO criteria:

Types of Studies

An observational study (cohort, nested case-control, case-control, etc.) that reported the outcome in a full-text version and had sufficient number of participants (we used a cutoff of $\geq 500$ participants). Study participants could be either gender with no restrictions in term of the region, ethnicity, use of other comedications, and comorbidities. 
Types of Participants

Adults (aged 18 years or greater) with any types of dementia with statin use and that was confirmed by the International Classification of Diseases, Ninth/Tenth revision, Diagnostic and Statistical Manual of Mental Disorders, 4th Edition (DSM-IV), DSM-III-R, Alzheimer's Association (NIA-AA) criteria for allcause dementia, National Institute of Neurological and Communicative Disorders and Stroke and the AD and Related Disorders Association clinical criteria (or both).

\section{Types of Interventions}

Patients who were using statin medication for at least 30 days versus patients who were not using statin medication.

Types of Outcome Measures

Primary outcomes: Overall risk of any kind of dementia with statin use.

Secondary outcomes: Development of $\mathrm{AD}$ and $\mathrm{VaD}$ in the patients with statin use.

\section{Data Extraction and Quality Assessment}

Two authors (T.N.P., M.M.I.) independently performed the search and extracted the data according to our standard guidelines. Each author entered the following data from each included study:

Methods

Study design, total duration of study, number of study centers and location, study setting, and date of study.

\section{Participants}

Number of participants, mean age, age range, gender, diagnosis at baseline, diagnostic criteria, inclusion criteria, and exclusion criteria.

Interventions

Intervention, comparison, and concomitant medication.

\section{Outcomes}

Primary and secondary outcomes.

We also noted the characteristics of the included studies and all variables used in these included studies. We resolved all disagreements by consensus or by involving our principle investigator (Y.-C.L.). Finally, we entered the data into the Review Manager software (RevMan: 5) and double-checked them for accuracy.

\section{Assessment of Risk of Bias in Included Studies}

We assessed the methodological quality of the included observational studies based on a modified version of the Newcastle-Ottawa Scale (NOS), which is recommended by the Cochrane Collaboration. NOS uses a star system (maximum of 9 stars) to assess a study in 3 areas which are (1) the selection of participants, (2) the comparability of the study groups, and (3) the ascertainment of the outcomes of interest. We categorized each study as a low risk, medium risk, or high risk if the study received a score of $9,7-8$, or $\leq 6$ stars, respectively.

\section{Statistical Analyses}

The outcome of interest of this meta-analysis was the dementia risk among individuals using statins compared with the dementia risk among individuals not using statins. We pooled the summary
RR with a 95\% CI from hazard ratios and ORs. Hazard ratio and ORs approximated RRs since dementia is a sufficiently rare event $(<15 \%$ per year), and most of the case-control studies use an open cohort sampling design. We used the DerSimonian and Laird random-effects model to pool the results which considered both within- and between study variation [20]. A risk ratio of $>1$ indicates an increased risk of dementia, and one of $<1$ indicates a decreased risk of dementia. We used forest plots to evaluate the possibility of statistical heterogeneity, and the $I^{2}$ value to assess statistical heterogeneity that provided an estimates of the percentage of variability among the included studies. An $I^{2}$ value of 25, 50, and 75\% represents low, medium, and high heterogeneity, respectively [21]. The results from all included studies were pooled, and an overall estimate of the effect size was determined using a random effect model that helps to reduce the heterogeneity among the studies [22]. The funnel plot and Egger regression test were used to evaluate publication bias. In addition, a sensitivity analysis was conducted due to the expected heterogeneity of the eligible studies. We also calculated subgroup differences based on the design (cohort and case-control), region (North America, Europe, and Asia), gender (male and female), duration of statin therapy (short, moderate, and long-term), and different class of statin use such as simvastatin, atorvastatin, pravastatin, and so on. All the statistical tests were two-tailed and used a significance level of $p$ value $\leq 0.05$. The comprehensive meta-analysis (CMA, V-2) was used for all statistical analyses.

\section{Results}

\section{Characteristics of Included Studies}

A total of 2,513 articles were identified in our initial search. Of these, 2,475 articles were excluded, and 38 potentially relevant studies were subjected to full-text screening. Only 30 observational studies fulfilled all our criteria and were therefore included in our meta-analysis [23-52]. Figure 1 shows the summary of excluded and included studies.

Table 1 summarizes the 30 eligible studies. These studies involved a total of 9,162,509 participants with 84,101 dementia patients from 23 cohort studies [23$25,29-42,45,46,48-51]$ and 7 case-control studies [26-28, 43, 44, 47, 52]. Of these, 20 studies were published in North America [24, 26, 33-36, 38-49, 51, 52], 4 studies in Europe [31, 32, 37, 50], and 6 studies in Asia $[23,25,27-30]$. The eligible studies confirmed dementia patients by using standard protocols such as International Classification of Diseases, Ninth/Tenth revision-CM, DSM-IV, DSM-III-R National Institute of Neurological and Communicative Disorders and Stroke and the $\mathrm{AD}$ and Related Disorders Association, ADAS-Cog, 3MSE, whereas statin users were identified by the Anatomical Therapeutic Chemical Classification System. Of the 30 included studies, 3 studies had high 
Fig. 1. PRISMA flow diagram for study selection.

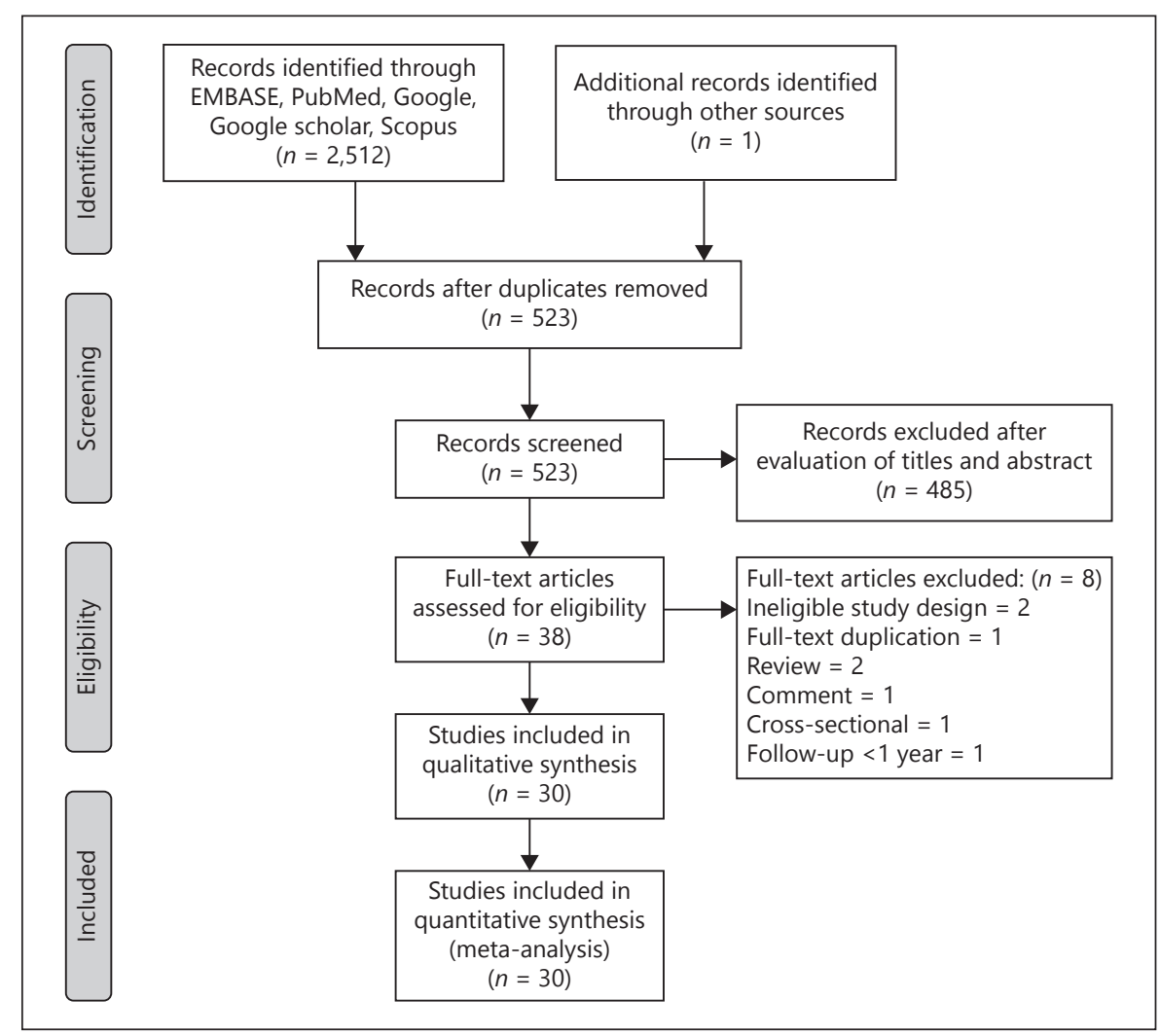

methodological quality (NOS 9), 25 studies had moderate quality (NOS 7-8), and 2 studies had low methodological quality (NOS $<7$ ).

\section{Primary Analysis}

Use of Statin and Dementia Risk

A total of 30 studies evaluated the association between statin use and all-caused dementia risk. Statin use was significantly associated with a decreased risk of dementia (RR 0.832, 95\% CI 0.793-0.872, $p<0.0001$ ). The overall heterogeneity among the studies was moderate $\left(I^{2}=\right.$ $\left.61.64, \tau^{2}=0.006, p<0.0001\right)$ in the random effect model (Fig. 2). Note that the fully adjusted value of each eligible study was considered for the pooled overall RR.

\section{Secondary Analyses}

Risk of AD with Statin Use

Twenty studies evaluated the association between statin use and the risk of AD. Statin use was again significantly associated with a decreased risk of AD. The overall pooled reduction of $\mathrm{AD}$ in patients with statin use was RR 0.69 (95\% CI $0.60-0.80, p<0.0001$ ) with a moderate heterogeneity $\left(I^{2}=47.59, \tau^{2}=0.041, p=0.010\right)$ in the random effect model (Fig. 3a).

\section{Use of Statin and VaD Risk}

Four studies evaluated the association between statin use and the risk of $\mathrm{VaD}$ risk. These 4 studies reported this risk separately from the risk associated with statin therapy. The overall pooled change of $\mathrm{VaD}$ demonstrated no significant association between statin use and $\mathrm{VaD}$ risk (RR $0.93,95 \%$ CI $0.74-1.16, p=0.54$ ) and showed moderate heterogeneity $\left(I^{2}=53.30, \tau^{2}=0.0611, p=0.004\right)$ in the random effect model (Fig. 3b).

\section{Subgroup Analysis}

We further stratified the analysis of statin use and dementia risk by study design, region, gender, duration of statin therapy, and different class of statin use. It is potentially important, however, that this may provide exact association by reducing bias and is needed for an establishment of their association (Table 2).

Twenty-three cohort studies evaluated the association between use of statin and dementia risk. The overall pooled RR was 0.84 (95\% CI 0.79-0.88), and the test of heterogeneity was moderate among the included studies $\left(I^{2}=54.58, \mathrm{Q}=48.78, \tau^{2}=0.004\right)$. Seven case-control studies evaluated the association of statin use and dementia risk. The overall pooled RR was 0.67 (95\% CI $0.55-$ 


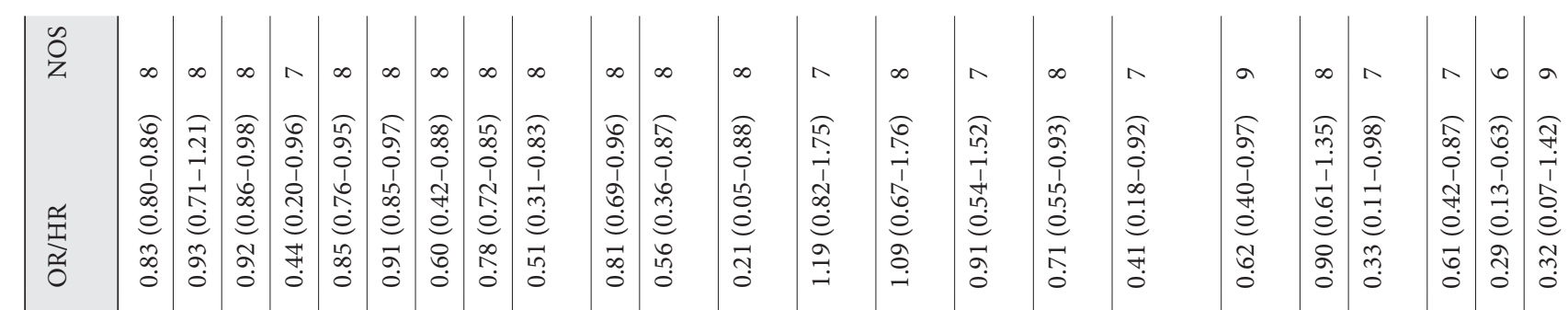

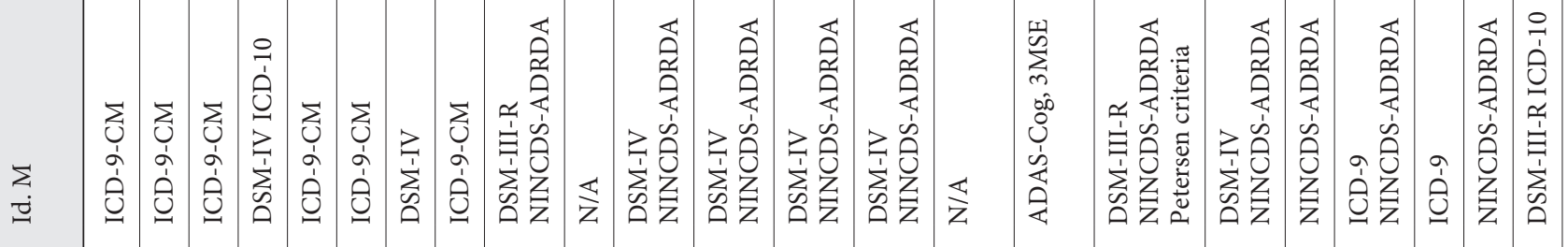

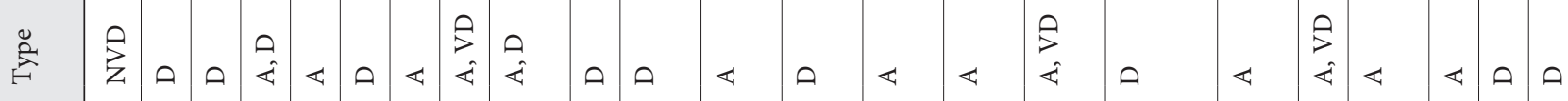

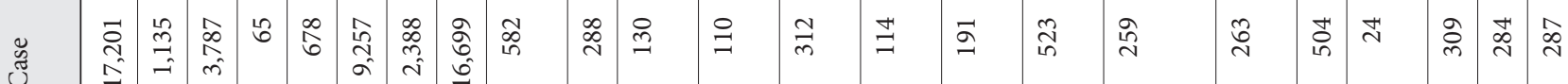

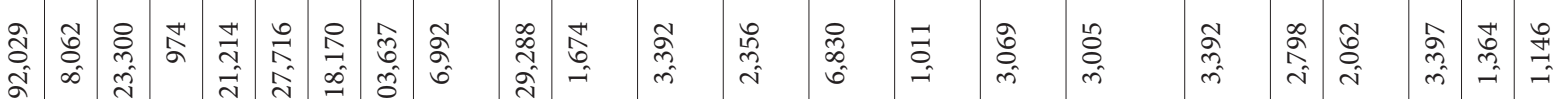

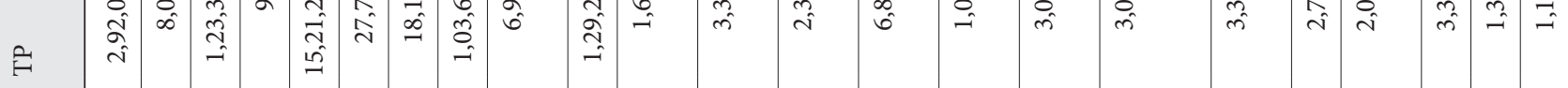

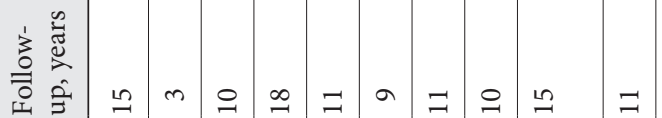

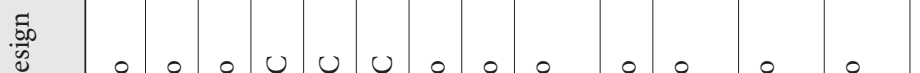

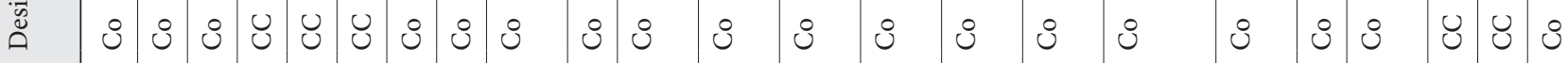

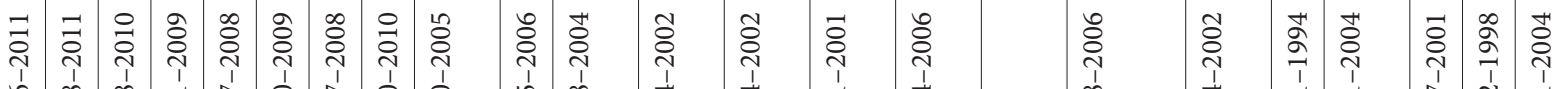

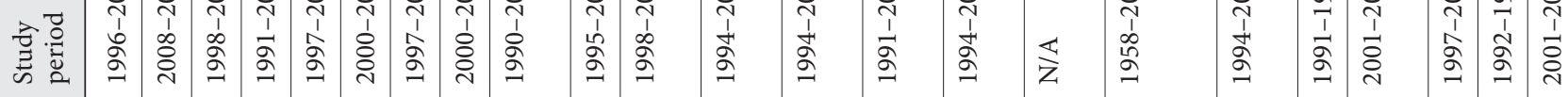

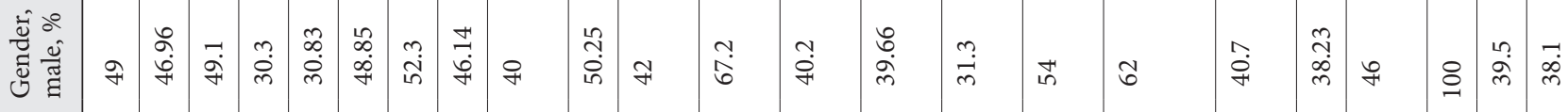

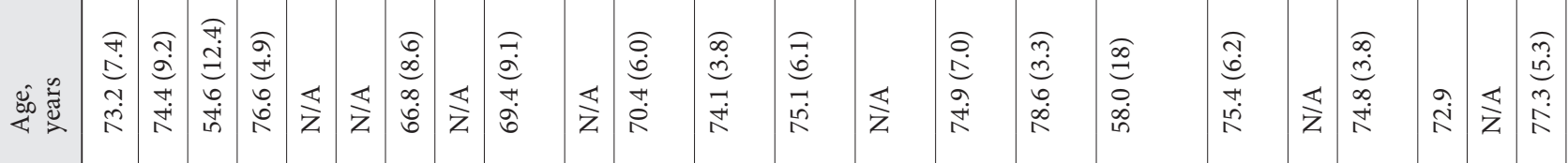

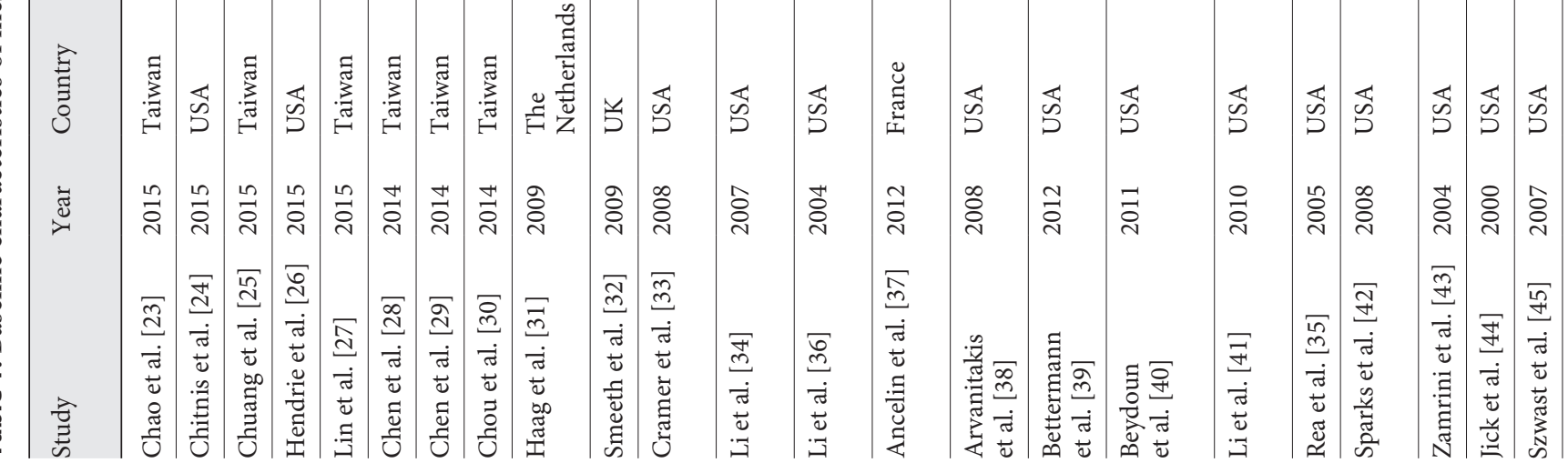




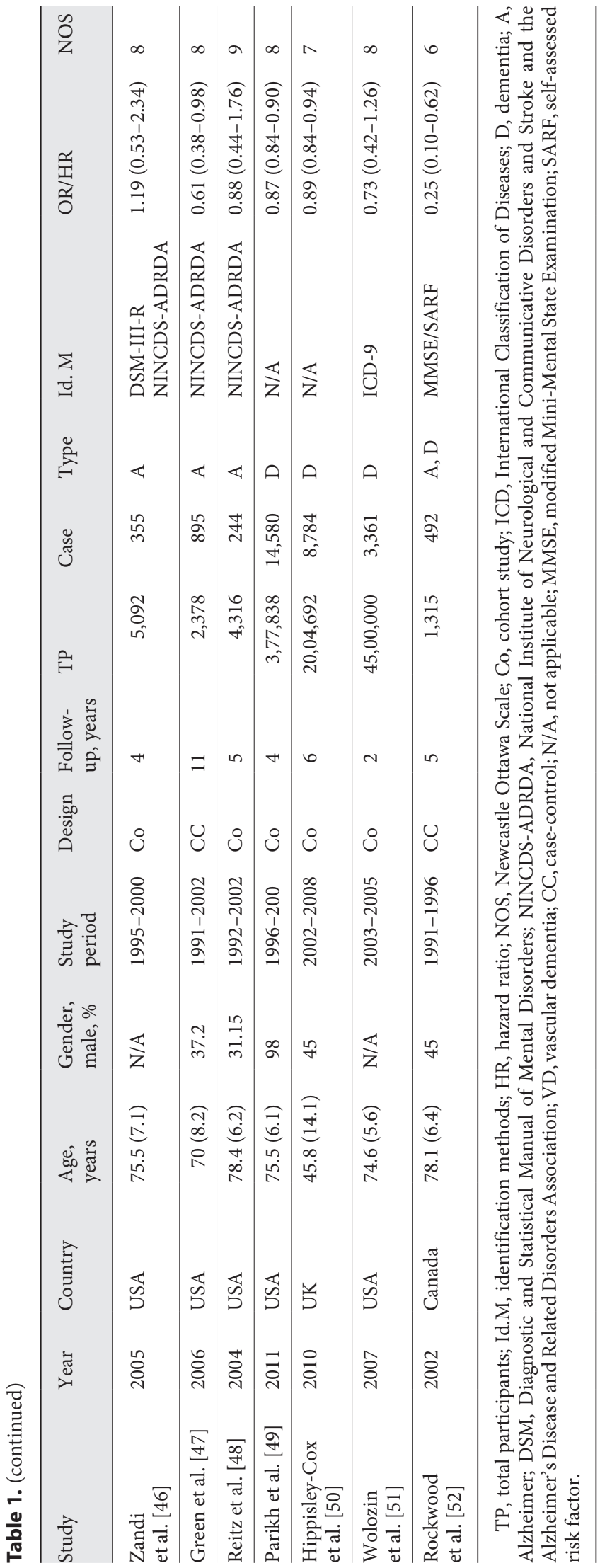

$0.83)$, and the test of heterogeneity was $\left(I^{2}=77.32, \mathrm{Q}=\right.$ $26.46, \tau^{2}=0.037$ ).

Five studies assessed dementia risk in male patients (RR 0.86 95\% CI 0.80-0.93), and 6 studies assessed dementia risk in female patients (RR 0.88 95\% CI 0.81$0.95)$. The pooled RR of dementia risk for studies from North America was 0.69 (95\% CI 0.60-0.81), for studies from Europe was 0.85 (95\% CI 0.74-0.99), and for studies from Asia was 0.85 (95\% CI 0.79-0.90).

Four studies evaluated the association between longterm statin use ( $>3$ years) and dementia risk. The overall pooled RR was RR 0.37 (95\% CI 0.19-0.73). Five studies also estimated dementia risk in patients with a medium-long exposure of statins ( $2-3$ years). The overall RR was 0.66 (95\% CI 0.52-0.83). However, the overall pooled RR for dementia risk in patients with a short duration of statin use was 1.04 (95\% CI $0.95-$ 1.13).

Four studies assessed the association between dementia risk and the use of different statin medications. The overall pooled RR for atorvastatin was 0.86 (95\% CI 0.760.98 ), for fluvastatin was 0.99 (95\% CI $0.90-1.10$ ), for lovastatin was 1.16 (95\% CI 0.93-1.33), for pravastatin was 0.91 (95\% CI $0.77-1.08)$, and for rosuvastatin was 0.59 (95\% CI 0.42-0.82). Five studies separately evaluated the effect of simvastatin. The overall pooled RR was 0.81 (95\% CI 0.54-1.21).

\section{Sensitivity Analysis}

Dementia risk was evaluated on the basis of statin potency. High potency statin was significantly associated with a decreased risk of dementia risk (RR 0.68, 95\% CI $0.54-0.86, p<0.001$ ), while low potency statin was not associated with a decreased risk of dementia (RR 0.92, $95 \%$ CI 0.87-0.97, $p=0.005$ ). In addition, 4 studies evaluated the risk of dementia with hydrophilic statin $[28,30$, $31,39]$, and 5 studies assessed the risk of dementia with lipophilic statin $[28,30,31,38,39]$. Hydrophilic statin decreased the dementia risk by $28 \%$ (RR $0.72,95 \%$ CI $0.63-0.82, p<0.0001$ ), and lipophilic statin decreased the dementia risk by $16 \%$ (RR $0.84,95 \%$ CI $0.75-0.94, p=$ $0.002)$.

\section{Publication Bias}

Visual inspection of the funnel plot was used to evaluate the possible publication bias. Figure 4 shows the existence of some publication bias. The Egger's regression test of the funnel asymmetry, however, showed no observed publication bias $(p<0.002)$. 


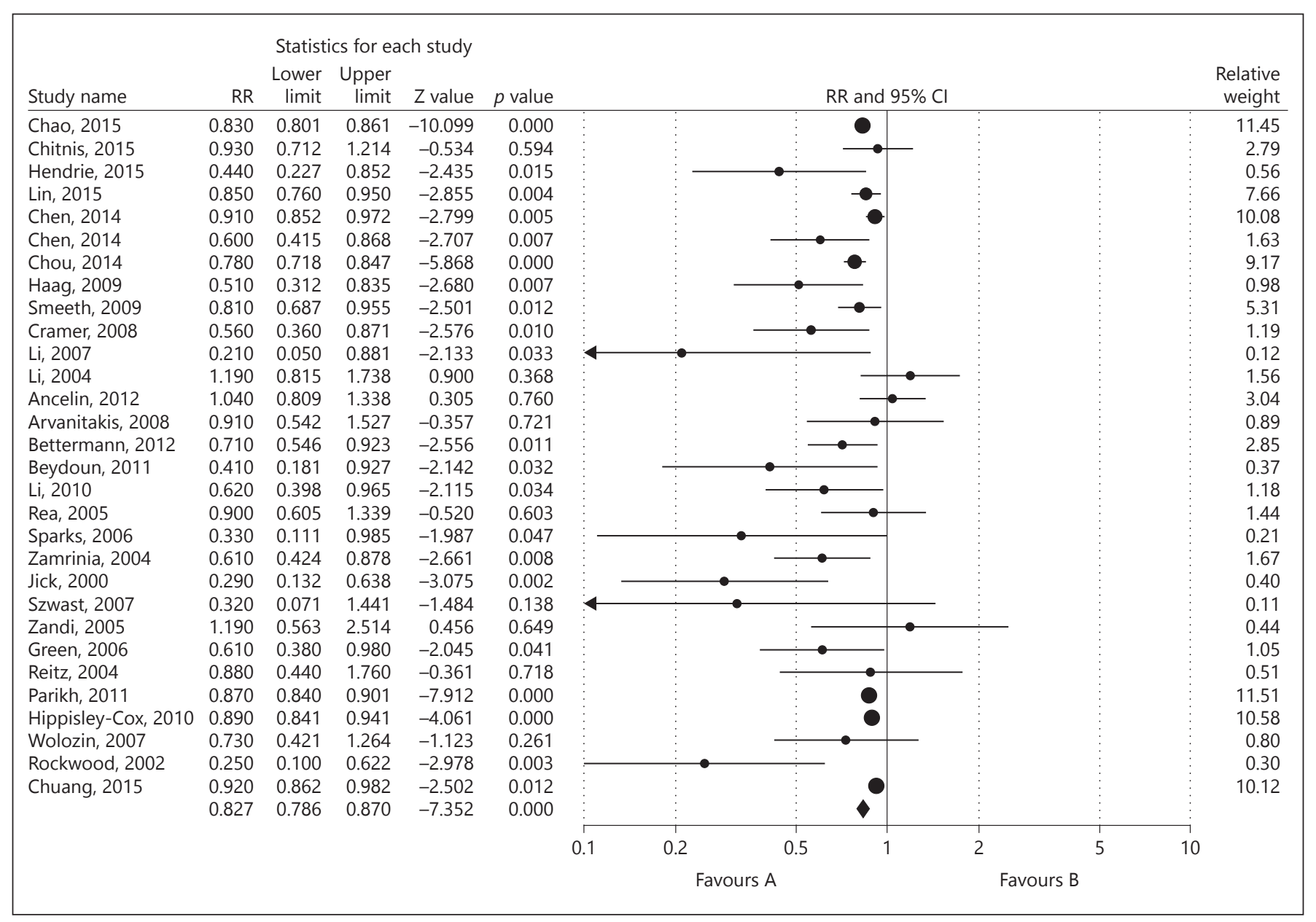

Fig. 2. Statin use and all-caused dementia risk. RR, risk ratio.

\section{Discussion}

\section{Main Findings}

Our meta-analysis provides evidence for an association between statin use and dementia risk. Our CMA included a total of 30 observational studies with aggregate data of $9,162,509$ individuals, of whom 84,101 were dementia patients. We found that statin use was associated with a $17 \%$ decreased risk of all-caused dementia. We also found that statin use was associated with a significantly decreased the risk of $\mathrm{AD}$ and neutral in $\mathrm{VaD}$. Our results are thus similar to those by 4 previously published meta-analyses $[10,14$, $53,54]$. The quality of the evidence was high because of our study design which only included studies with higher methodological quality and lower bias and findings of our study are fully adjusted in age group, gender, and different types of covariates (e.g., stroke, diabetics, hypertension, ischemic heart disease, cerebrovascular disease, etc.).
When the analysis was stratified by gender, the risk of dementia was decreased both in male and female patients with statins. However, North American individuals had a lower risk compared with Europeans and Asians. Furthermore, baseline characteristics of each study were provided that helped to reduce the confounding factors of our current meta-analysis. Unfortunately, most of the previously studies lacked subgroup analyses and used only a low number of studies. Ours is the largest and most updated meta-analysis aimed at quantifying the magnitude of the association between statins and dementia risk. Notably, our meta-analysis is the first to show that the association between different types of statins (namely, rosuvastatin, atorvastatin, and simvastatin) and dementia risk. Moreover, we also considered the duration of statin use in order to make our study more robust, and statin use of longer duration ( $>3$ years) affected a significantly decreased risk of dementia. 


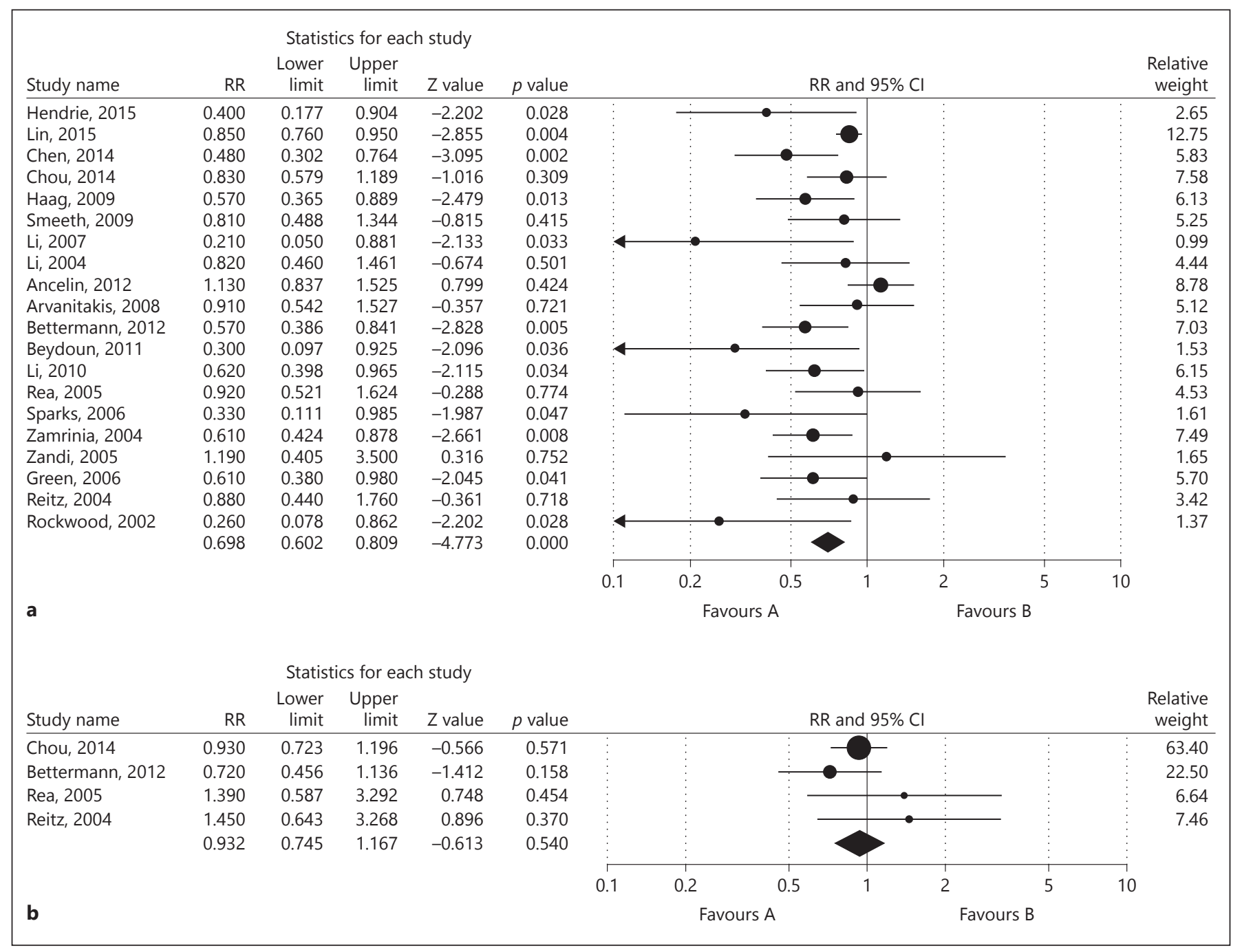

Fig. 3. a Statin use and $\mathrm{AD}$ risk. b Statin use and $\mathrm{VaD}$ risk. $\mathrm{RR}$, risk ratio.

\section{Biological Evidence}

There are several possible biological mechanisms that might explain the association between statin use and dementia risk. Previous studies suggested some specific mechanisms of statin action that could help to reduce dementia risk (Table 3 ). First, the potent anti-inflammatory properties of statins are involved in inducing the endothelial nitric oxide synthesis, which is crucial for vascular function and maintenance [55]. Endothelial nitric oxide synthase augmentation is linked to increased cerebral vasomotor reactivity and cerebral blood flow regulation [56] and improved neurovascular dysfunction. Indeed, the neurovascular dysfunction reversal is associated with the improvement of cognitive function. Second, the regulation of low lipid lipoprotein oxidation by statins might play an important role in dementia prevention by the reduction of atherosclerosis [57]. Experimental and clinical studies have revealed a relationship between tumor necrosis factor-alpha and the causation of AD. Statins significantly suppress tumor necrosis factor-alpha synthesis in microglia and reduce neuronal damage [58]. Finally, the extent of neuronal loss and the risk of dementia correlate with the production of amyloid- $\beta$ [59] (Fig. 5). These various possible mechanisms suggest that statins have preventive properties against dementia.

\section{Clinical Implication}

Nowadays, statins treatments for the prevention of dementia have been gained lots of attention. Researchers and medical professional have been evaluated the poten- 
Table 2. Subgroup analysis regarding statins use and dementia risk

\begin{tabular}{|c|c|c|c|c|c|c|c|c|}
\hline Study & \multicolumn{3}{|l|}{ Pool estimate } & \multicolumn{4}{|c|}{ Test of heterogeneity } & Model \\
\hline \multicolumn{9}{|l|}{ Study design } \\
\hline Case-control & 7 & $0.67(0.55-0.83)$ & $<0.0001$ & 77.32 & 26.46 & 0.037 & $<0.0001$ & $\mathrm{RE}$ \\
\hline Cohort & 23 & $0.84(0.79-0.88)$ & $<0.0001$ & 54.98 & 48.87 & 0.004 & 0.001 & $\mathrm{RE}$ \\
\hline Women & 6 & $0.88(0.81-0.95)$ & 0.001 & 65.30 & 14.41 & 0.005 & 0.013 & $\mathrm{RE}$ \\
\hline \multicolumn{9}{|l|}{ Region } \\
\hline North America & 20 & $0.69(0.60-0.81)$ & $<0.0001$ & 60.11 & 47.63 & 0.047 & $<0.0001$ & $\mathrm{RE}$ \\
\hline Europe & 4 & $0.85(0.74-0.99)$ & 0.03 & 60.11 & 7.52 & 0.012 & 0.057 & $\mathrm{RE}$ \\
\hline Asia & 6 & $0.85(0.79-0.90)$ & $<0.0001$ & 73.58 & 18.92 & 0.004 & 0.002 & $\mathrm{RE}$ \\
\hline \multicolumn{9}{|l|}{ Individual statins } \\
\hline Rosuvastatin & 4 & $0.59(0.42-0.82)$ & 0.002 & 96.47 & 85.09 & 0.094 & $<0.0001$ & $\mathrm{RE}$ \\
\hline Simvastatin & 5 & $0.81(0.54-1.21)$ & 0.324 & 99.57 & 930.05 & 0.203 & $<0.001$ & $\mathrm{RE}$ \\
\hline \multicolumn{9}{|l|}{ Duration, years } \\
\hline$\leq 1$ & 3 & $1.04(0.95-1.13)$ & 0.35 & 27.24 & 2.74 & 0.002 & 0.253 & $\mathrm{RE}$ \\
\hline $2-3$ & 5 & $0.66(0.52-0.83)$ & $<0.0001$ & 81.78 & 21.96 & 0.044 & $<0.0001$ & $\mathrm{RE}$ \\
\hline$>3$ & 4 & $0.37(0.19-0.73)$ & 0.004 & 99.50 & 602.17 & 0.43 & $<0.0001$ & $\mathrm{RE}$ \\
\hline
\end{tabular}

$\mathrm{RR}$, risk ratio; $\mathrm{RE}$, random effect.

Fig. 4. Funnel plot. RR, risk ratio.

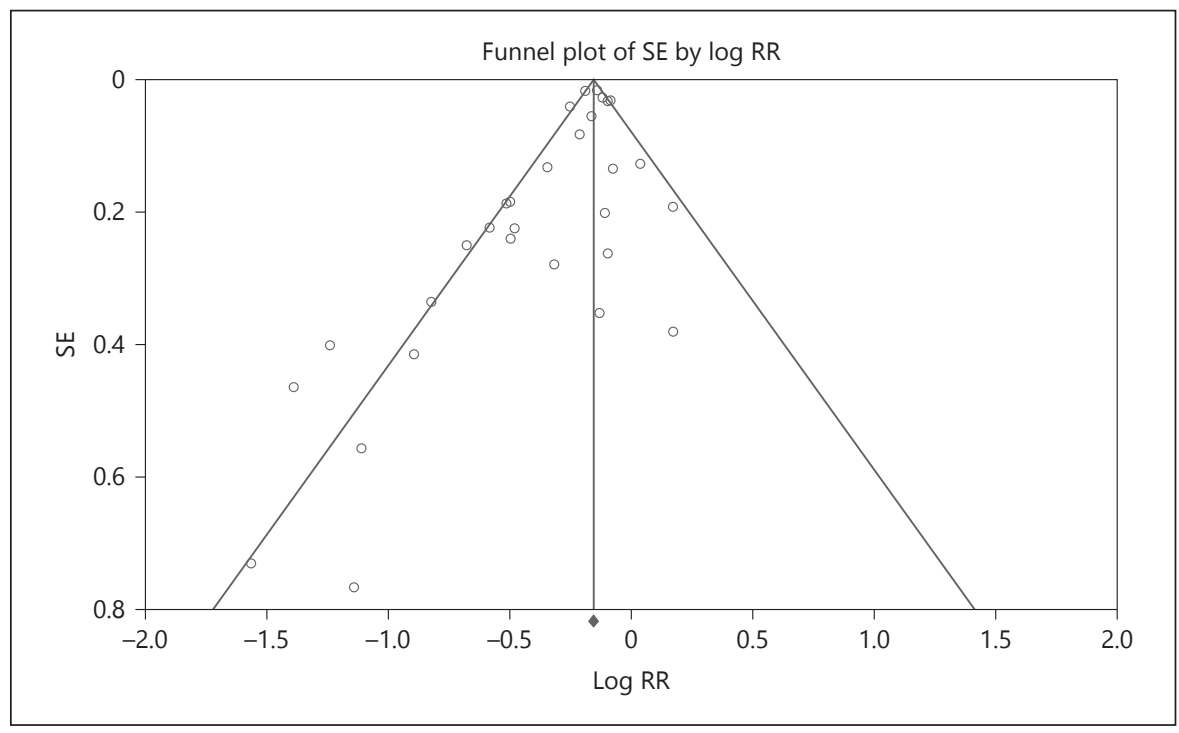

tial beneficial effects of statins treatment on dementia or cognitive risk reduction. Several systematic review and meta-analysis of epidemiological studies have been examined the protective effects of statins treatments [12, 60]. Patients with statins had a significantly lower risk of developing all-type of dementia when compared with patients without statins. Biologically, it seems admissible that statins reduce the risk of dementia due to their lipidlowering effect instead of other mechanism. Lower cholesterol levels in the midlife help to reduce the risk of all 
Table 3. Beneficial action of statin on dementia

\begin{tabular}{llll}
\hline Clinical evidence & & Targets of statins & isoprenoid pathway \\
\cline { 3 - 4 } $\begin{array}{l}\text { Oxidative stress and } \\
\text { inflammatory stress }\end{array}$ & $\begin{array}{l}\text { Oxidative stress and inflammatory } \\
\text { stress are involved in AD }\end{array}$ & & $\begin{array}{l}\text { Rac1-NADPH and PPAR- } \alpha,-\gamma \\
\text { and NF- } \kappa \mathrm{B}\end{array}$ \\
\hline Vascular system & $\begin{array}{l}\text { Midlife vascular risk factors } \\
\text { potentiate development of AD }\end{array}$ & $\begin{array}{l}\text { Cholesterol-induced } \\
\text { cerebrovascular atherosclerosis }\end{array}$ & PI3K/Akt/eNOS pathway \\
\hline Tau accumulation & $\begin{array}{l}\text { Tau starts to accumulate decades } \\
\text { before the onset of AD }\end{array}$ & $\begin{array}{l}\text { Cholesterol-induced tau hyper } \\
\text { phosphorylation }\end{array}$ & $\begin{array}{l}\text { Cholesterol-independent } \\
\text { anti-inflammatory effects }\end{array}$ \\
\hline A $\beta$ accumulation & $\begin{array}{l}\text { A } \beta \text { starts to accumulate decades } \\
\text { before the onset of AD }\end{array}$ & $\begin{array}{l}\text { Cholesterol-involving A } \beta \\
\text { production }\end{array}$ & $\begin{array}{l}\text { APP-CTFs degradation via Rho } \\
\text { or Rab family, and A } \beta \text { clearance } \\
\text { via LRP1 or IDE }\end{array}$ \\
\hline
\end{tabular}

$\mathrm{AD}$, Alzheimer's disease; Rac1, Ras-related C3 botulinum toxin substrate 1; NADPH, nicotinamide adenine dinucleotide phosphate; PPAR, peroxisome proliferator-activated protein; NF- $\kappa$ B, nuclear factor- $\kappa \mathrm{B}$; PI3K, phosphatidylinositol-4,5-bisphosphate3-kinase; Akt, $\mathrm{v}$-akt murine thymoma viral oncogene homolog; eNOS, endothelial nitric oxide synthase; A $\beta$, $\beta$-amyloid peptides; APP-CTFs, C-terminal fragments of amyloid precursor protein; LRP1, LDLR-related protein 1; IDE, insulin degrading enzyme.

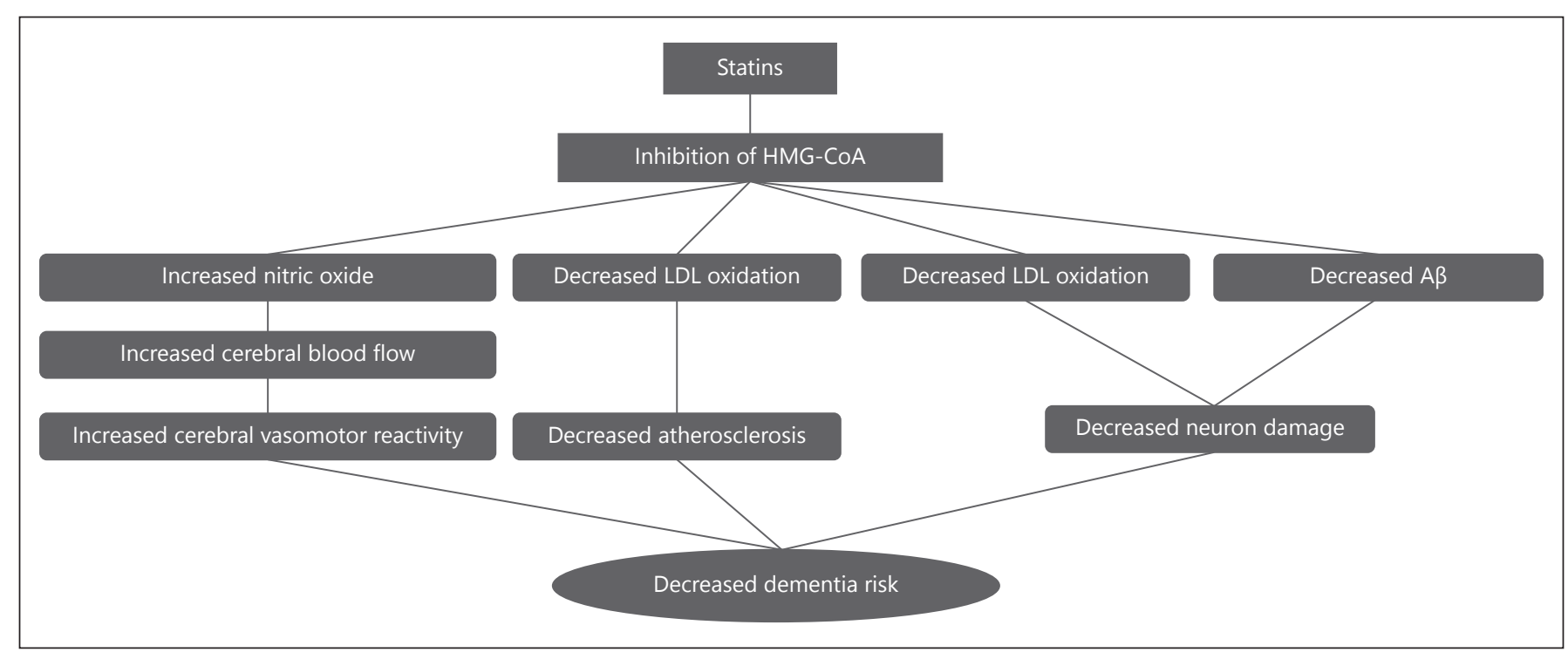

Fig. 5. Possible biological effects of decreasing dementia risk by statins. HMG-CoA, $\beta$-hydroxy $\beta$-methylglutaryl-CoA; LDL, low-density lipoprotein; $A \beta$, amyloid- $\beta$.

types of dementia in late-life. Power et al. [61] reported that higher level of total cholesterol in mild-life was associated with increased risk of late-life dementia compared to normal cholesterol.

However, given the widespread use of statins and their enormous benefits in hypercholesterolemia, the American College of Cardiology and the American Heart Association provided guidelines to prescribe moderate- or high-inten- sity statin therapy if the patients fulfill certain criteria. A moderate intensity (standard) of statin therapy helps to reduce low-density lipoprotein (LDL) cholesterol by 30 $50 \%$, while a high intensity (strong) statin therapy helps to reduce LDL by $50 \%$ or more. Our results showed that strong statins, such as rosuvastatin, atorvastatin, and simvastatin, were associated with a larger reduction of dementia, whereas standard statins, such as pravastatin, fluvas- 
tatin, and lovastatin, were not associated with a reduction of dementia. Therefore, the potency of statin played a crucial role in reducing the risk of dementia. The lipophilicity of statin prevents tissue distribution that also alters the pharmacological and clinical effects [62]. Our study shows that the greatest risk reduction in dementia was associated with the hydrophilic statins (e.g., rosuvastatin). Longer exposure times ( $>2$ years) were associated with an even greater risk reduction in dementia. Since North American patients had a higher reduction rate compared to European and Asian people, diet (saturated fats, trans fats, and cholesterol), life styles, and genetic factors may also play important roles. Furthermore, statin therapy could be influenced by other medical conditions, such as hypertension, diabetics, atrial fibrillation, and stroke; however, adjustments for these risk factors in our study did not change the effect size of the association between statin therapy and risk of dementia. The findings of our meta-analysis provide an appropriate, critical, and responsible approach to evaluate the association between statin use and reduced risk of dementia; no matter how many confounding factors and systematic errors of bias or methodological weakness are among these included studies. Healthcare providers and clinical policymakers should take these findings seriously; statins should probably be prescribed as a promise treatment for patients with AF aged $\geq 50$ years to prevent all kinds of dementia in addition to lowering lipid levels.

The current evidence regarding the association between statin use and dementia risk is predominantly based on retro/prospective observational studies. Most epidemiologic studies can only suggest associations, which are prone to various observed biases leading to a possibly erroneous extrapolation to causality. However, no study design is perfect and conflicting finding could be emerging from all types of studies [15]. Furthermore, the pooled estimate from included large observational studies with moderate follow-up is tailored to minimize bias. Additionally, there is no single, best approach, and there are no exact hard and fast rules that can reasonably judge causation. Clinical and public health decision making from the findings of observational studies (patients typical of day to day clinical care data) would help to improve the quality of healthcare while reducing the rising cost [63]. However, the findings from meta-analysis of observational studies with reasonable biological hypotheses can provide valid evidence for clinical and public health action. In modern day to day clinical practice, RCT-based clinical information is scarce and lacks the potential of a heterogeneous population. Alongside RCT studies, meta-analyses are generating useful information from large interven- tional epidemiological studies and providing new opportunities as a complementary source of information to gain stronger and more reliable evidence for treatment action $[64,65]$. Furthermore, researchers have to find out the appropriate answer to questions like: what effect has the long-term use of these medications in patients who may not need chronic therapy? And what to do for a patient who truly needs chronic statins therapy?

\section{Strengths and Limitations}

Our meta-analysis has several strengths. First, it is the first meta-analysis that provides a comprehensive overview of statin use and dementia risk from 30 observational studies. Second, this study included 9.1 million individuals with 84,101 dementia patients who are an accurate reflection of dementia patients commonly seen in routine clinical practice. Therefore, our study provides a trustworthy result that statin use significantly reduces the risk of dementia. Third, our study examines the association in great detail, as we stratified our analysis by study design, regional effects, characteristics of statin, duration, and individual statin exposure. Finally, visual inspection of the funnel plot and formal tests demonstrated no observed statistical evidence of publication bias in this meta-analysis. Notwithstanding these strengths, our study also has some limitations that need to be mentioned. The random effect model was used to assess the heterogeneity among the included studies, and we observed a moderate $(57.73 \%)$ heterogeneity in the overall primary analysis. This moderate heterogeneity was due to the demographic and ethno-racial characteristics of the study populations, sample size, study design, follow-up period, and in the magnitude of statin use and dementia risk. Furthermore, some important variables, such as different comorbidity, medication, BMI, and smoking status, which may impact dementia risk, were missing in our subgroup analyses because of the lack of data. Finally, our findings based on observational studies may trigger "false alarms" due to poorly adjusted study parameters applied to a retro/prospectively analysis [66]. This study has focused on reporting pooled summary estimates and draws conclusions from included eligible observational studies. Despite providing more comprehensive and precise findings, this study cannot offer experimental evidence regarding the causal nature of the reported associations.

\section{Recommendation for Future Research}

A substantial number of studies have been published on statin use and its associated dementia risk over the past few years. Despite this large number of publications, there 
is room for improvement to our understanding of the probable biological mechanisms. To address these issues, we should:

1. Conduct more high-quality longitudinal studies with longer follow-up periods, which include both new and chronic statins users. Such studies would help to reduce selection bias and potential confounding variables.

2. The biological causal mechanisms between statin use and dementia risk reduction are not well established. Therefore, we need more translational research as well as clinical studies to understand the fundamental biological causes, which may explain the observed association.

3. We need to better measure the levels of exposure to potential confounding variables, for example, comedication medication exposure, dose, duration, and date of initiating (never and ever use category). Additionally, information about comorbidity chronicity, severity, and treatment requirement would be useful.

\section{Conclusion}

Our CMA showed that statin use is significantly associated with a decreased risk of dementia. Considering the evidence presented in our meta-analysis, it is feasible that statins can contribute to prevent dementia risk because of their role in high total cholesterol reduction. Moreover, the results of our meta-analysis should open a path for future large observational studies with long follow-up periods. Our findings highlight that population-based data on the association of statin use and dementia risk reduction remain limited, and that further study (e.g., RCT, biological study) into the exact role in the etiology of dementia risk reduction is warranted.

\section{Disclosure Statement}

None.

\section{References}

1 Williams JW, Plassman BL, Burke J, Benjamin S. Preventing Alzheimer's disease and cognitive decline. Evid Rep Technol Assess (Full Rep). 2010 Apr;193(193):1-727.

2 Prince MJ, Wu F, Guo Y, Gutierrez Robledo LM, O'Donnell M, Sullivan R, et al. The burden of disease in older people and implications for health policy and practice. Lancet. 2015 Feb;385(9967):549-62.

3 Baumgart M, Snyder HM, Carrillo MC, Fazio S, Kim H, Johns H. Summary of the evidence on modifiable risk factors for cognitive decline and dementia: A population-based perspective. Alzheimers Dement. 2015 Jun;11(6):718-26.

4 Wang Y-C, Tai P-A, Poly TN, Islam MM, Yang H-C, Wu C-C, Li Y-CJ. Increased risk of dementia in patients with antidepressants: A meta-analysis of observational studies. Behav Neurol. 2018 Jul;2018:5315098.

5 Pahan K. Lipid-lowering drugs. Cell Mol Life Sci. 2006 May;63(10):1165-78.

6 Kurinami H, Sato N, Shinohara M, Takeuchi D, Takeda S, Shimamura M, et al. Prevention of amyloid $\beta$-induced memory impairment by fluvastatin, associated with the decrease in amyloid $\beta$ accumulation and oxidative stress in amyloid $\beta$ injection mouse model. Int $\mathrm{J} \mathrm{Mol}$ Med. 2008 May;21(5):531-7.

7 Tong XK, Nicolakakis N, Fernandes P, Ongali B, Brouillette J, Quirion R, et al. Simvastatin improves cerebrovascular function and counters soluble amyloid-beta, inflammation and oxidative stress in aged APP mice. Neurobiol Dis. 2009 Sep;35(3):406-14.

8 Thal DR, Griffin WS, de Vos RA, Ghebremedhin E. Cerebral amyloid angiopathy and its relationship to Alzheimer's disease. Acta Neuropathol. 2008 Jun;115(6):599-609.

9 Iadecola C. Neurovascular regulation in the normal brain and in Alzheimer's disease. Nat Rev Neurosci. 2004 May;5(5):347-60.

10 Zhou B, Teramukai S, Fukushima M. Prevention and treatment of dementia or Alzheimer's disease by statins: a meta-analysis. Dement Geriatr Cogn Disord. 2007;23(3):194-201.

11 Song Y, Nie H, Xu Y, Zhang L, Wu Y. Association of statin use with risk of dementia: a meta-analysis of prospective cohort studies. Geriatr Gerontol Int. 2013 Oct;13(4):817-24.

12 Wong WB, Lin VW, Boudreau D, Devine EB. Statins in the prevention of dementia and Alzheimer's disease: a meta-analysis of observational studies and an assessment of confounding. Pharmacoepidemiol Drug Saf. 2013 Apr; 22(4):345-58.

13 Rojas-Fernandez C, Hudani Z, Bittner V. Statins and cognitive side effects: what cardiologists need to know. Cardiol Clin. 2015 May;33(2):245-56.

14 Chu CS, Tseng PT, Stubbs B, Chen TY, Tang $\mathrm{CH}, \mathrm{Li} \mathrm{DJ}$, et al. Use of statins and the risk of dementia and mild cognitive impairment: A systematic review and meta-analysis. Sci Rep. 2018 Apr;8(1):5804.

15 Frieden TR. Evidence for health decision making - beyond randomized, controlled trials. N Engl J Med. 2017 Aug;377(5):465-75.

16 Islam MM, Yang HC, Nguyen PA, Poly TN, Huang CW, Kekade S, et al. Exploring association between statin use and breast cancer risk: an updated meta-analysis. Arch Gynecol Obstet. 2017 Dec;296(6):1043-53.
17 Islam MM, Iqbal U, Walther B, Atique S, Dubey NK, Nguyen PA, et al. Benzodiazepine use and risk of dementia in the elderly population: a systematic review and metaanalysis. Neuroepidemiology. 2016;47(3-4): 181-91.

18 Poly TN, Islam MM, Walther BA, Yang HC, Nguyen PA, Huang CW, et al. Exploring the association between statin use and the risk of Parkinson's disease: a meta-analysis of observational studies. Neuroepidemiology. 2017; 49(3-4):142-51.

19 Poly TN, Islam MM, Yang HC, Wu CC, Li YJ. Proton pump inhibitors and risk of hip fracture: a meta-analysis of observational studies. Osteoporos Int. 2019 Jan;30(1):103-14.

20 DerSimonian R, Laird N. Meta-analysis in clinical trials. Control Clin Trials. 1986 Sep; 7(3):177-88.

21 Higgins JP, Thompson SG, Deeks JJ, Altman DG. Measuring inconsistency in meta-analyses. BMJ. 2003 Sep;327(7414):557-60.

22 Higgins JP, Thompson SG, Spiegelhalter DJ. A re-evaluation of random-effects meta-analysis. J R Stat Soc Ser A Stat Soc. 2009 Jan; 172(1):137-59.

23 Chao TF, Liu CJ, Chen SJ, Wang KL, Lin YJ, Chang SL, et al. Statins and the risk of dementia in patients with atrial fibrillation: A nationwide population-based cohort study. Int J Cardiol. 2015 Oct;196:91-7.

24 Chitnis AS, Aparasu RR, Chen H, Kunik ME, Schulz PE, Johnson ML. Use of statins and risk of dementia in heart failure: a retrospective cohort study. Drugs Aging. 2015 Sep; 32(9):743-54. 
25 Chuang CS, Lin CL, Lin MC, Sung FC, Kao $\mathrm{CH}$. Decreased prevalence of dementia associated with statins: a national populationbased study. Eur J Neurol. 2015 Jun;22(6): 912-8.

26 Hendrie HC, Hake A, Lane K, Purnell C, Unverzagt F, Smith-Gamble V, et al. Statin use, incident dementia and Alzheimer disease in elderly African Americans. Ethn Dis. 2015 Aug;25(3):345-54.

27 Lin FC, Chuang YS, Hsieh HM, Lee TC, Chiu $\mathrm{KF}$, Liu CK, et al. Early statin use and the progression of Alzheimer disease: a total population-based case-control study. Medicine (Baltimore). 2015 Nov;94(47):e2143.

28 Chen PY, Liu SK, Chen CL, Wu CS. Longterm statin use and dementia risk in Taiwan. J Geriatr Psychiatry Neurol. 2014 Sep;27(3): $165-71$.

29 Chen JM, Chang CW, Chang TH, Hsu CC, Horng JT, Sheu WH. Effects of statins on incident dementia in patients with type $2 \mathrm{DM}$ : a population-based retrospective cohort study in Taiwan. PLoS One. 2014 Feb;9(2):e88434.

30 Chou CY, Chou YC, Chou YJ, Yang YF, Huang N. Statin use and incident dementia: a nationwide cohort study of Taiwan. Int J Cardiol. 2014 May;173(2):305-10.

31 Haag MD, Hofman A, Koudstaal PJ, Stricker $\mathrm{BH}$, Breteler MM. Statins are associated with a reduced risk of Alzheimer disease regardless of lipophilicity. The Rotterdam Study. J Neurol Neurosurg Psychiatry. 2009 Jan;80(1):13-

32 Smeeth L, Douglas I, Hall AJ, Hubbard R, Evans $\mathrm{S}$. Effect of statins on a wide range of health outcomes: a cohort study validated by comparison with randomized trials. Br J Clin Pharmacol. 2009 Jan;67(1):99-109.

33 Cramer C, Haan MN, Galea S, Langa KM Kalbfleisch JD. Use of statins and incidence of dementia and cognitive impairment without dementia in a cohort study. Neurology. 2008 Jul;71(5):344-50.

34 Li G, Larson EB, Sonnen JA, Shofer JB, Petrie EC, Schantz A, et al. Statin therapy is associated with reduced neuropathologic changes of Alzheimer disease. Neurology. 2007 Aug 69(9):878-85.

35 Rea TD, Breitner JC, Psaty BM, Fitzpatrick AL, Lopez OL, Newman AB, et al. Statin use and the risk of incident dementia: the Cardiovascular Health Study. Arch Neurol. 2005 Jul; 62(7):1047-51.

36 Li G, Higdon R, Kukull WA, Peskind E, Van Valen Moore K, Tsuang D, et al. Statin therapy and risk of dementia in the elderly: a community-based prospective cohort study. Neurology. 2004 Nov;63(9):1624-8.

37 Ancelin ML, Carrière I, Barberger-Gateau P, Auriacombe S, Rouaud O, Fourlanos S, et al. Lipid lowering agents, cognitive decline, and dementia: the three-city study. J Alzheimers Dis. 2012;30(3):629-37.
38 Arvanitakis Z, Schneider JA, Wilson RS, Bienias JL, Kelly JF, Evans DA, et al. Statins, incident Alzheimer disease, change in cognitive function, and neuropathology. Neurology. 2008 May;70(19 Pt 2):1795-802.

39 Bettermann K, Arnold AM, Williamson J, Rapp S, Sink K, Toole JF, et al. Statins, risk of dementia, and cognitive function: secondary analysis of the ginkgo evaluation of memory study. J Stroke Cerebrovasc Dis. 2012 Aug; 21(6):436-44

40 Beydoun MA, Beason-Held LL, Kitner-Triolo MH, Beydoun HA, Ferrucci L, Resnick SM, et al. Statins and serum cholesterol's associations with incident dementia and mild cognitive impairment. J Epidemiol Community Health. 2011 Nov;65(11):949-57.

41 Li G, Shofer JB, Rhew IC, Kukull WA, Peskind ER, McCormick W, et al. Age-varying association between statin use and incident $\mathrm{Al}$ zheimer's disease. J Am Geriatr Soc. 2010 Jul; 58(7):1311-7.

42 Sparks DL, Sabbagh M, Connor D, Soares H, Lopez J, Stankovic G, et al. Statin therapy in Alzheimer's disease. Acta Neurol Scand Suppl. 2006;185:78-86.

43 Zamrini E, McGwin G, Roseman JM. Association between statin use and Alzheimer's disease. Neuroepidemiology. 2004 Jan-Apr; 23(1-2):94-8

44 Jick H, Zornberg GL, Jick SS, Seshadri S, Drachman DA. Statins and the risk of dementia. Lancet. 2000 Nov;356(9242):1627-31.

45 Szwast SJ, Hendrie HC, Lane KA, Gao S, Taylor SE, Unverzagt F, et al. Association of statin use with cognitive decline in elderly African Americans. Neurology. 2007 Nov;69(19): 1873-80.

46 Zandi PP, Sparks DL, Khachaturian AS, Tschanz J, Norton M, Steinberg M, et al.; Cache County Study investigators. Do statins reduce risk of incident dementia and $\mathrm{Al}$ zheimer disease? The Cache County Study. Arch Gen Psychiatry. 2005 Feb;62(2):217-24.

47 Green RC, McNagny SE, Jayakumar P, Cupples LA, Benke K, Farrer LA; MIRAGE Study Group. Statin use and the risk of Alzheimer's disease: the MIRAGE study. Alzheimers Dement. 2006 Apr;2(2):96-103.

48 Reitz C, Tang MX, Luchsinger J, Mayeux R. Relation of plasma lipids to Alzheimer disease and vascular dementia. Arch Neurol. 2004 May;61(5):705-14.

49 Parikh NM, Morgan RO, Kunik ME, Chen H, Aparasu RR, Yadav RK, et al. Risk factors for dementia in patients over 65 with diabetes. Int J Geriatr Psychiatry. 2011 Jul;26(7):749-57.

50 Hippisley-Cox J, Coupland C. Unintended effects of statins in men and women in England and Wales: population based cohort study using the QResearch database. BMJ. 2010 May; 340:c2197.

51 Wolozin B, Wang SW, Li NC, Lee A, Lee TA, Kazis LE. Simvastatin is associated with a re- duced incidence of dementia and Parkinson's disease. BMC Med. 2007 Jul;5(1):20.

52 Rockwood K, Kirkland S, Hogan DB, MacKnight $\mathrm{C}$, Merry $\mathrm{H}$, Verreault R, et al. Use of lipid-lowering agents, indication bias, and the risk of dementia in community-dwelling elderly people. Arch Neurol. 2002 Feb;59(2): 223-7.

53 Zhang X, Wen J, Zhang Z. Statins use and risk of dementia: A dose-response meta analysis. Medicine(Baltimore).2018Jul;97(30):e11304.

54 Zhu XC, Dai WZ, Ma T. Overview the effect of statin therapy on dementia risk, cognitive changes and its pathologic change: a systematic review and meta-analysis. Ann Transl Med. 2018 Nov;6(22):435.

55 Zhou Q, Liao JK. Pleiotropic effects of statins. - Basic research and clinical perspectives Circ J. 2010 May;74(5):818-26.

56 Giannopoulos S, Katsanos AH, Tsivgoulis G, Marshall RS. Statins and cerebral hemodynamics. J Cereb Blood Flow Metab. 2012 Nov; 32(11):1973-6.

57 Yarchoan M, Xie SX, Kling MA, Toledo JB, Wolk DA, Lee EB, et al. Cerebrovascular atherosclerosis correlates with Alzheimer pathology in neurodegenerative dementias. Brain. 2012 Dec;135(Pt 12):3749-56.

58 Birkenhager W, Cheung B, Kumana C, Jick H, Blauw G, Kast R, et al. Dementia and statins. LANCET-LONDON; 2001. pp. 880-1.

59 Vaughan CJ. Prevention of stroke and dementia with statins: effects beyond lipid lowering. Am J Cardiol. 2003 Feb;91(4 4A):23B9B.

60 Shah NP, Swiger KJ, Martin SS. Impact on cognitive function-are all statins the same? Curr Atheroscler Rep. 2015 Jan;17(1):466.

61 Power MC, Rawlings A, Sharrett AR, Bandeen-Roche K, Coresh J, Ballantyne CM, et al. Association of midlife lipids with 20-year cognitive change: A cohort study. Alzheimers Dement. 2018 Feb;14(2):167-77.

62 Hamelin BA, Turgeon J. Hydrophilicity/lipophilicity: relevance for the pharmacology and clinical effects of HMG-CoA reductase inhibitors. Trends Pharmacol Sci. 1998 Jan;19(1): 26-37.

63 Langham S, Langham J, Goertz HP, Ratcliffe M. Large-scale, prospective, observational studies in patients with psoriasis and psoriatic arthritis: A systematic and critical review. BMC Med Res Methodol. 2011 Mar;11(1):32.

64 Gopalakrishnan S, Ganeshkumar P. Systematic reviews and meta-analysis: understanding the best evidence in primary healthcare. J Family Med Prim Care. 2013 Jan;2(1):9-14.

65 Angus DC. Fusing randomized trials with big data: the key to self-learning health care systems? JAMA. 2015 Aug;314(8):767-8.

66 Grimes DA, Schulz KF. False alarms and pseudo-epidemics: the limitations of observational epidemiology. Obstet Gynecol. 2012 Oct;120(4):920-7. 